\title{
Pulmonary hypertension: a contraindication to the use of $\beta$-adrenoceptor blocking agents
}

\author{
A Peacock, K Ross
}

Scottish Pulmonary Vascular Unit, Level 1 Regional Heart and Lung Centre, Golden Jubilee National Hospital, Glasgow, UK

\section{Correspondence to}

A Peacock, Scottish Pulmonary Vascular Unit, Level 1 Regional Heart and Lung Centre, Golden Jubilee National Hospital, Beardmore Street, Glasgow G81 $4 \mathrm{HX}, \mathrm{UK}$

apeacock@udcf.gla.ac.uk

Received 2 June 2009

Accepted 12 January 2010

\section{ABSTRACT}

Supraventricular tachycardia (SVT) is a rare but important complication of pulmonary arterial hypertension. $\beta$-Adrenoceptor blocking agents are commonly used to treat SVT, but they are potentially dangerous in the context of pulmonary arterial hypertension. Their negative inotropic and chronotropic effects are poorly tolerated in this condition, where cardiac reserve is compromised by a reduced and fixed stroke volume, and their administration can result in significant cardiorespiratory compromise. This is not a new discovery, but it is nonetheless poorly recognised in day-to-day clinical practice.

\section{CASE HISTORY}

The patient presented with portopulmonary hypertension secondary to alcoholic liver disease diagnosed 2 years previously by echocardiography and cardiac catheterisation (mean pulmonary artery pressure $40 \mathrm{~mm} \mathrm{Hg}$, pulmonary capillary wedge pressure $6 \mathrm{~mm} \mathrm{Hg}$, pulmonary vascular resistance $11.3 \mathrm{WU}$, cardiac index $1.9 \mathrm{l} / \mathrm{min} / \mathrm{m}^{2}$ ). She had a background of alcohol excess and liver cirrhosis for which she had undergone a transjugular intrahepatic portosystemic shunt procedure 15 years earlier. No further factors were identified that could have contributed to the pulmonary hypertension and her only other medical history of note was a recent pulmonary embolism, treated with 3 months of warfarin, and episodic supraventricular tachycardia (SVT).

She had been discharged from the Scottish Pulmonary Vascular Unit (SPVU) 1 week previously following a review admission. Her clinical condition had remained relatively stable since her diagnosis but she had dyspnoea and a decision was made to add sildenafil. She was taking sildenafil $50 \mathrm{mg}$ three times daily and bosentan $62.5 \mathrm{mg}$ twice daily, a reduced dose due to the introduction of sildenafil.

Within $48 \mathrm{~h}$ of discharge from the SPVU she developed symptoms of increasing dyspnoea, vomiting, periorbital and upper limb swelling, leftsided chest pain and abdominal distension. Four days later she presented to a district general hospital emergency department with saturations of $92 \%$ on 101 oxygen, heart rate 94 beats/min and blood pressure $147 / 92 \mathrm{~mm} \mathrm{Hg}$. She was dyspnoeic with clear lungs, cool peripheries, oedema and a raised jugular venous pressure. Arterial blood gas analysis on $10 \mathrm{l}$ oxygen showed $\mathrm{H}^{+} 42 \mathrm{nmol} / \mathrm{l}, \mathrm{PO}_{2}$ $7.8 \mathrm{kPa}, \mathrm{PCO}_{2} 2.95 \mathrm{kPa}$, base excess $-10.6 \mathrm{mmol} / 1$ and lactate $7.6 \mathrm{mmol} / \mathrm{l}$, consistent with hypoxia and a partially compensated metabolic acidosis. A chest x-ray showed borderline cardiomegaly and the ECG showed sinus rhythm with right axis deviation and partial left bundle branch block. Initial biochemistry revealed D-dimer $1173 \mathrm{ng} / \mathrm{ml}$ (normal value $<500 \mathrm{ng} / \mathrm{ml}$ ) and deranged liver function tests (bilirubin $29 \mu \mathrm{mol} / 1$, alkaline phosphatase $178 \mathrm{IU} / \mathrm{l}, \boldsymbol{\gamma}$-glutamyl transpeptidase $240 \mathrm{U} / \mathrm{l})$.

Following discussion with the SPVU, sildenafil was discontinued, bosentan was increased back to $125 \mathrm{mg}$ daily and treatment for pulmonary thromboembolism was administered pending CT pulmonary angiography.

Late in the evening the patient developed SVT. During this episode she struggled to maintain saturations of $80 \%$ on high-flow oxygen and had a heart rate of approximately 175 beats $/ \mathrm{min}$. A repeat chest x-ray was unchanged. Administration of adenosine restored sinus rhythm and stabilised the vital signs. Treatment with amiodarone was started and, following consultation with the cardiology department regarding potentially ongoing tachyarrythmias, a single dose of $25 \mathrm{mg}$ oral metoprolol was administered. There was no evidence of left ventricular impairment.

Within approximately $30 \mathrm{~min}$ the patient was in peri-arrest. Saturations dipped to $50 \%$ on high-flow oxygen with a heart rate of 59 beats/min and blood pressure $68 / 40 \mathrm{~mm} \mathrm{Hg}$. Arterial blood gas analysis showed significant hypoxaemia and a mixture of metabolic acidosis and respiratory alkalosis $\left(\mathrm{H}^{+}\right.$ $30.5 \mathrm{nmol} / 1, \mathrm{PO}_{2} 4.45 \mathrm{kPa}, \mathrm{PCO}_{2} 3.5 \mathrm{kPa}$, lactate $3.4 \mathrm{mmol} / \mathrm{l}$ ). A repeat ECG showed new lateral Twave inversion. The sympathomimetic $\beta$-adrenergic agonist isoprenaline was commenced and vital signs slowly started to stabilise.

Approximately $4 \mathrm{~h}$ later, following a further recurrence of sustained SVT, the isoprenaline was stopped. Other than one self-terminating episode of bradycardia, no further arrhythmias developed. The CT pulmonary angiogram was negative for pulmonary thromboembolism and repeat echocardiography was essentially unchanged compared with 6 months previously, showing a dilated right heart and poor right ventricular function with severe tricuspid regurgitation. The raised D-dimer level is most likely explained by the combination of liver disease and congestive cardiac failure.

Two days later the patient was stable and was transferred to the SPVU where she had an uneventful stay. With the most likely explanation for her hospital admission being the introduction of sildenafil, this remained discontinued and she was discharged home on her original regime of bosentan $125 \mathrm{mg}$ twice daily. 


\section{DISCUSSION}

Pulmonary arterial hypertension (PAH) is a recognised complication of portal hypertension secondary to cirrhosis. ${ }^{1}$ Although rare, it is a cause of significant morbidity and mortality. Patients exhibit extensive pulmonary vascular remodelling, increased pulmonary vascular resistance and right heart failure. It is diagnosed against a background of cirrhosis with elevated right ventricular pressures on echocardiography, and confirmed by right heart catheterisation which demonstrates mean pulmonary artery pressure $>25 \mathrm{~mm} \mathrm{Hg}^{2}$

The potential detrimental effects of $\beta$-adrenoceptor blockers in PAH are poorly recognised in day-to-day clinical practice. Indeed, our review of the literature yielded just one directly relevant paper. ${ }^{3}$ This study observed the effect of $\beta$-blocker withdrawal at 2 months in patients with $\mathrm{PAH}$ receiving $\beta$-blockers for prophylaxis of variceal bleeding. It reported improvements in pulmonary haemodynamics, exercise capacity, 6-min walking distance and cardiac output, with decreased pulmonary vascular resistance.

$\beta$-Blockers are competitive antagonists of the effect of catecholamines at $\beta$-adrenoceptor sites with resultant negative inotropic and chronotropic effects. They are generally well tolerated and adverse effects are transient but, in sensitive patients or following larger doses, severe cardiovascular depression has been reported. ${ }^{4-6}$ Adverse effects include deterioration in right ventricular function, severe hypotension, bradycardia, increased ventilation-perfusion mismatch and hypoxaemia. In $\mathrm{PAH}$, where cardiac reserve is already compromised by a reduced and fixed stroke volume, the additional chronotropic depression imposed by $\beta$-blockers can result in significant or, indeed, fatal cardiovascular compromise.

When $\beta$-blockers are used in combination with other antiarrhythmic agents or drugs affecting cardiac conduction, the potential for precipitating bradycardia and heart block is increased. Bradycardia, cardiac arrest and ventricular fibrillation have all been reported on starting $\beta$-blockers in patients receiving amiodarone. ${ }^{7}$ Of note, amiodarone has been shown to increase plasma concentrations of metoprolol. ${ }^{8}$

SVT is an important but rare complication of PAH secondary to right-sided atrial and ventricular enlargement as a result of chronic pressure overload. A single-centre study by Tongers et al found it to have a cumulative incidence of $11.7 \%$ and an annual risk of $2.8 \%$ per patient. ${ }^{9}$ Its onset is generally associated with right ventricular failure and results in marked clinical deterioration. The final clinical outcome varies with different types of SVT. Atrial flutter and atrioventricular nodal re-entry tachycardia are more likely to be restored to sinus rhythm with appropriate therapeutic intervention, whereas atrial fibrillation, if sustained, has a high associated mortality.

Treatment options for SVT in this context are interventional or medical. Interventional approaches include overdrive pacing, radiofrequency ablation and electrical cardioversion. Although effective, these require adequate specialised equipment, staffing and training, and are not generally an option at 03:00 in a district general hospital. Medical options include adenosine, amiodarone, the calcium channel antagonist verapamil, digoxin and $\beta$-adrenoceptor blockers. Each has its drawbacks. While adenosine and verapamil are commonly used to treat SVT in the emergency department, the former is associated with a significant incidence of unpleasant side effects and the latter can result in hypotension. ${ }^{10}$ Digoxin has a narrow therapeutic index and amiodarone is associated with a host of adverse effects, notably interstitial lung disease and significant liver injury, especially after prolonged usage.

As acknowledged by Tongers et al, there is a lack of evidence to fully understand the incidence, consequences and optimal treatment options for SVTs in PAH.

In conclusion, in an attempt to control SVT, a rare but important complication of $\mathrm{PAH}$, our patient was given a small dose of a short-acting $\beta_{1}$-adrenoceptor blocking agent, the effects of which may have been potentiated by the simultaneous administration of amiodarone. The cardiovascular depression provoked was not tolerated by a heart impaired by portopulmonary hypertension. In the setting of a fixed reduced stroke volume, suppression of the chronotropic response resulted in insuffient cardiac output to maintain clinical stability.

Competing interests None.

Patient consent Obtained.

Provenance and peer review Not commissioned; externally peer reviewed.

\section{REFERENCES}

1. Rodriguez-Roisin R, Krowka MJ, Herve P, et al. ERS Task Force Pulmonary-Hepatic Vascular Disorders Scientific Committee. Pulmonary-hepatic vascular disorders. Eur Respir J 2004;24:861-80.

2. Blei AT. Portal hypertension and its complications. Curr Opin Gastroenterol 2007;23:275-82

3. Provencher S, Herve P, Xavier J, et al. Deleterious effects of beta-blockers on exercise capacity and haemodynamics in patients with portopulmonary hypertension. Gastroenterology 2006;130:120-6.

4. Tirlapur VG, Evans PJ, Jones MK, et al. Shock syndrome after acebutolol. Br J Clin Pract 1986; 40:33-4.

5. Kholeif M, Isles C. Profound hypotension after atenolol in severe hypertension. BMJ 1989;298:161-2

6. Lewis M, Kallenbach J, Germond C, et al. Survival following massive overdose of adrenergic blocking agents (acebutolol and labetalol). Eur Heart J 1983;4:328-32.

7. Lesko LJ. Pharmacokinetic drug interactions with amiodarone. Clin Pharmacokinet 1989;17:130-40.

8. Fukumoto $\mathbf{K}$, Kobayashi T, Tachibana $\mathrm{K}$, et al. Effect of amiodarone on the serum concentration/dose ratio of metoprolol in patients with cardiac arrhythmia. Drug Metab Pharmacokinet 2006;21:501-5.

9. Tongers J, Schwerdtfeger B, Klein G, et al. Incidence and clinical relevance of supraventricular tachyarrythmias in pulmonary hypertension. Am Heart $J$ 2006;153:127-32.

10. Holdgate A, Foo A. Intervention review: adenosine versus intravenous calcium channel antagonists for the treatment of supraventricular tachycardia in adults. Cochrane Database of Syst Rev 2006;(4):CD005154. 